\title{
Atomic Force Microscopy and Scanning Tunneling Microscopy of
}

\author{
Aluminum Nanoislands \\ S. Nedilko, V. Prorok, S. Rozouvan* \\ Department of Physics, Taras Shevchenko Kiev University, Glushkova 2, Kyiv, Ukraine \\ *e-mail: sgr@univ.kiev.ua
}

Keywords: aluminum nanoislands, scanning tunnelling microscopy, volt-ampere curves.

\begin{abstract}
Aluminum nanoislands deposited on silicon substrate were studied by a scanning tunnelling microscopy technique. Measurements completed with spatial resolution up to $1 \mathrm{~nm}$ revealed a complex nanoisland structure- rhomboidally ordered near the border of the aluminumsilicon and porous structure in the island's upper parts. Volt-ampere curves demonstrated strong dependency from the film thickness and were interpreted as nanoscale effects. Kelvin probe microscopy was used for simultaneous aluminum-alumina and alumina-air surface scanning.
\end{abstract}

\section{Introduction}

Scanning atomic force microscopy and scanning tunneling microscopy were proven to be extremely useful techniques for thin film's characterization, on metal or semiconductor substrate [1]. The technique allows researchers to obtain the films' valuable characteristic properties of inter-atomic distance spatial resolution level. Since recent nanotechnological advances, many research groups have intensely studied the nanoisland films [2]. 
Aluminum and alumina film's properties are especially interesting because the aluminum together with copper, gold and aluminum-copper alloys is an essential part of most modern electronic devices. The current research with alumina thin films are mostly conducted in a few main directions. The important part of the aluminum film's problems are related to alumina layers on the bulk aluminum. The layers have a higher protective mechanical and electrical parameters which are the key factors of the bulk aluminum properties. This seems to be the reason of the variety of studies with alumina films. For example, scanning tunnelling microscopy revealed particularities of crystalic lattice structure [3]. Data of Numerical simulations lead to understanding the ultrafast oxidation processes of aluminum (i.e. in time frames of a few tens picoseconds) [4] or during longer transition times [5]. Alumina film properties have been studied theoretically, particularly its' crystal lattice structure modification types [6], or connections between the lattice types [7]. Alumina-aluminum properties have been also numerically analyzed from the point of view of contacts between the alumina lattices and a metal [8] or between the layers with different level of ordering $[9,10]$.

Aluminum films properties have been studied mostly by applying scanning tunneling microscopy or electron microscopy techniques. For example, a hexagonal atomic structure was found in aluminum films [11-13]. Scanning tunneling microscopy was applied for investigation of plasmons in the contact aluminum-alumina [14]. Atomic force microscopy, which has a comparatively low spatial resolution has been applied in order to study the stability of the formation of the aluminum nanoislands $[15,16]$, or deposited aluminum film thermo-expansion as a function of electrical current [17].

The goal of this research is to further study aluminum island films deposited on silica by atomic force microscopy and scanning tunneling microscopy techniques. This will take us further inside of these nano materials. 


\section{Experiments and set-up description}

Aluminum islands films on silica substrate were thermally deposited in vacuum. The equipment is directly installed in the vacuum chamber which allowed controlling of the aluminum temperature, deposition speed and substrate temperature. The substrates which have been used for the film deposition were carefully cleaned and polished silica plates (the roughness was about 4-6 nm). The deposition time interval was of a few seconds, and the pressure in the vacuum chamber reached $10^{-5}$ torr during the deposition process. A microscope INTEGRA NT-MDT, allowed us to conduct the measurements in atomic force microscopy and tunneling microscopy regimes was used for precise surface topology study. Atomic force microscopy measurements have been conducted both in contact and semi-contact regimes. The measurements' spatial resolution has been determined by the cantilever tip curvature and reached $40 \mathrm{~nm}$ in horizontal direction. Scanning tunneling microscopy spatial resolution reached up to 1 nanometer.

\section{Results and discussion}

Aluminum nanoislands film surface topography obtained by atomic force microscopy semi-contact measurements are presented in Fig.1 (The film was obtained by thermal deposition during 20 seconds). The islands' size, visible in the image varies distinctly from tens to a few hundred nanometers. The data points out a complicated island nanoscale topology. In order to study the islands' structure more carefully, we used scanning tunneling microscopy technique. 


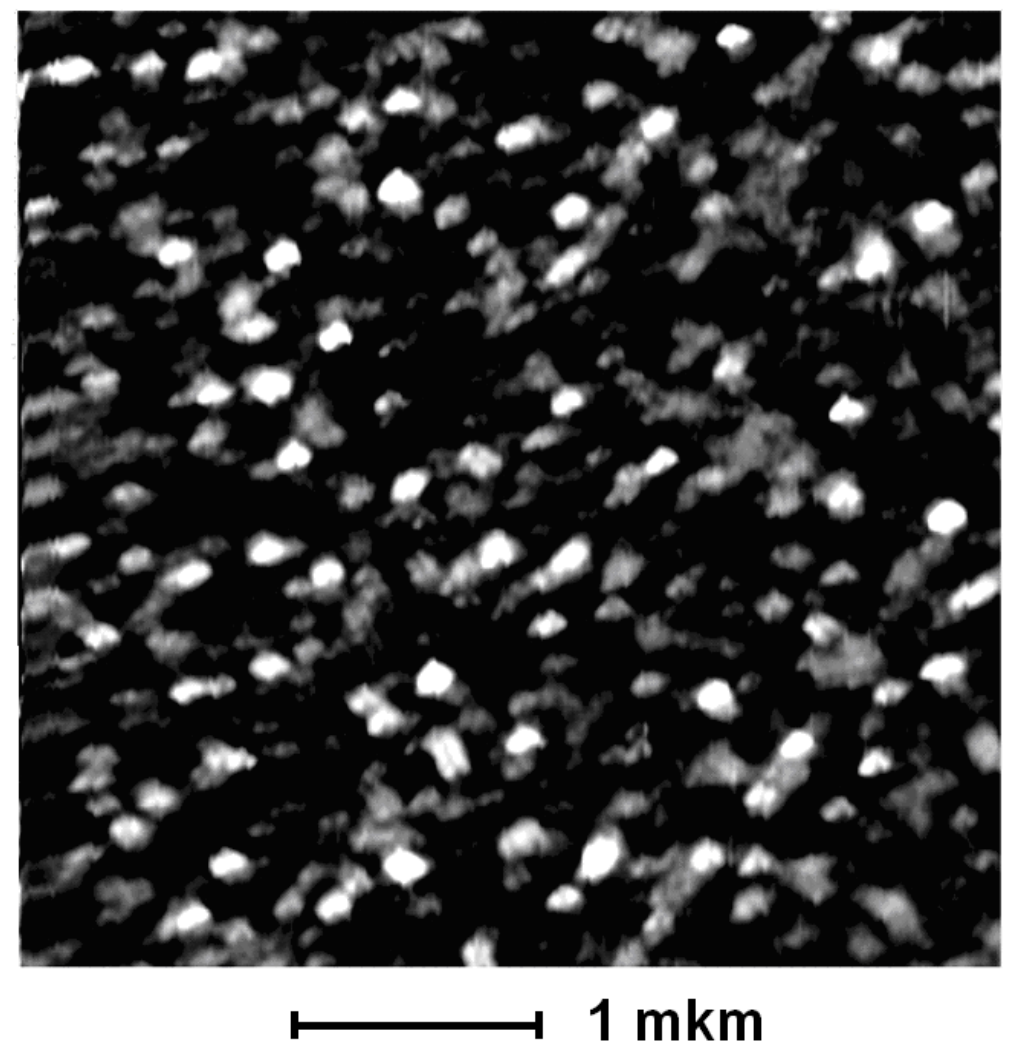

Fig. 1. Atomic force microscopy scan of surface of aluminum islands films on silica substrate.
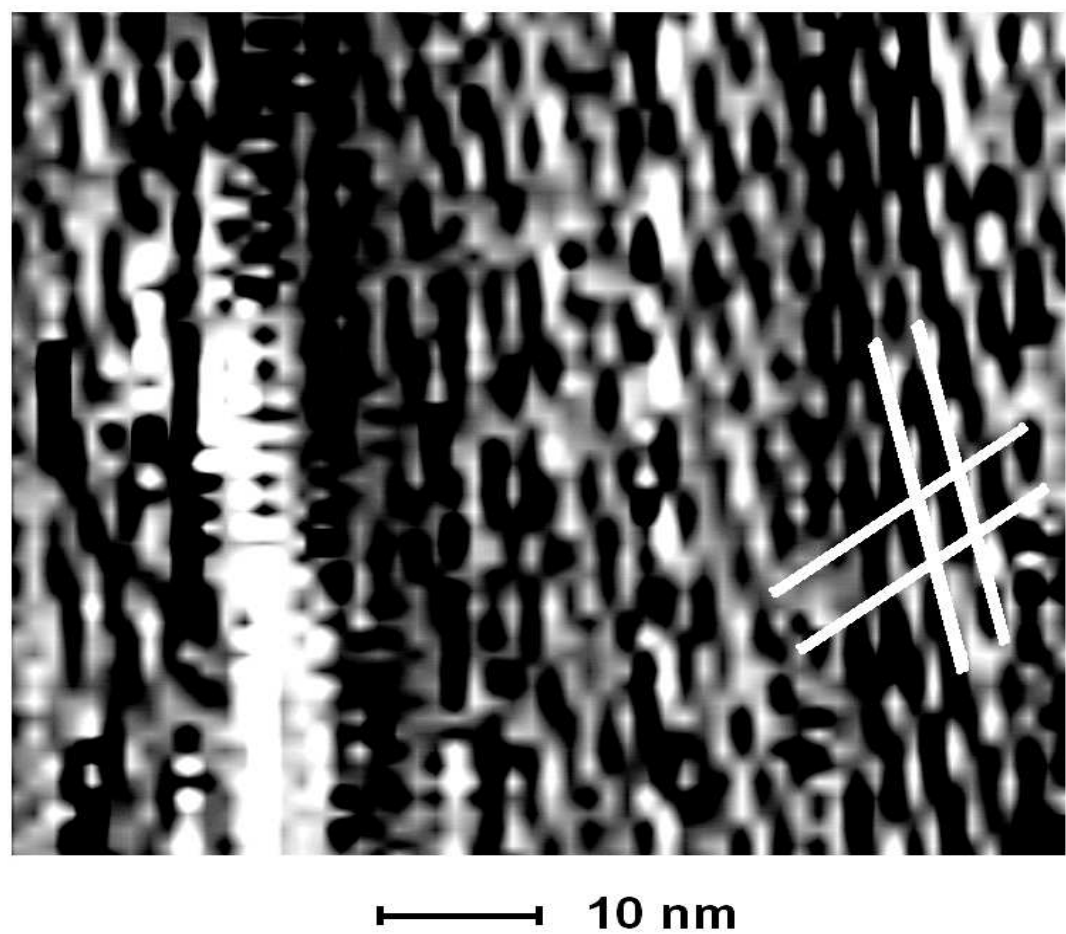

Fig. 2. Scanning tunneling microscopy scan of the surface of the aluminum island on silica substrate. Spatial resolution is $1 \mathrm{~nm}$. 
Typical scan results with space resolution of $1 \mathrm{~nm}$ are presented in Fig. 2. First of all, the dark background of the picture, which has lower electrical conductivity, comparing with neighboring regions, is originated from silica substrate. Secondly, the distinct rhomboidal gray structure (additionally marked by four white straight lines) with the height above the substrate roughness seems to be formed by alumina thin layer, each block of its rhombohedral structure (with darker inner parts) contains $7 \times 7$ unit $\mathrm{Si}$ lattice cells as it was shown in pioneer scanning tunneling microscopy studies [18]. The $\mathrm{Al}$ atoms are deposited and forming the nanoislands bottom layer with the rhomboidal pattern along Si top layer atoms without unsatisfied dangling bonds. The porous aluminum monoatomic layers exist just near the substrate surface and its' rhomboidal shape is influenced by silica face centered cubic lattice. Finally, the film structure in the places with higher thickness has nonrhomboidally ordered porous structure. The latter structure seems to be a typical for thicker aluminum films.

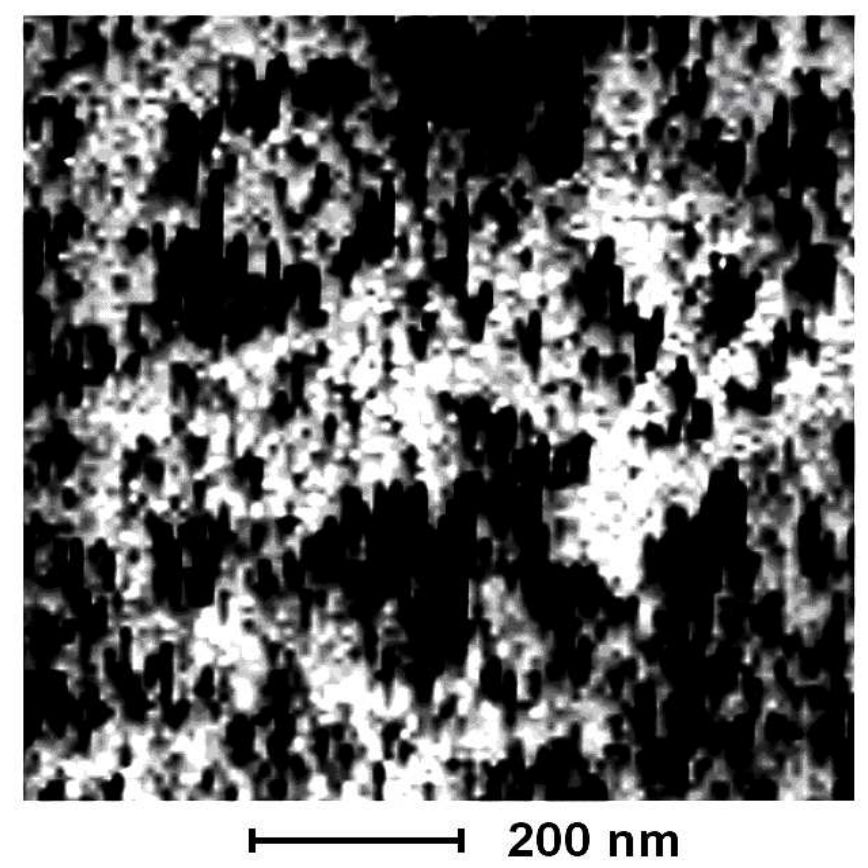

Fig. 3. Scanning tunneling microscopy scan of surface of aluminum islands' films on silica substrate. Spatial resolution is $5 \mathrm{~nm}$. 


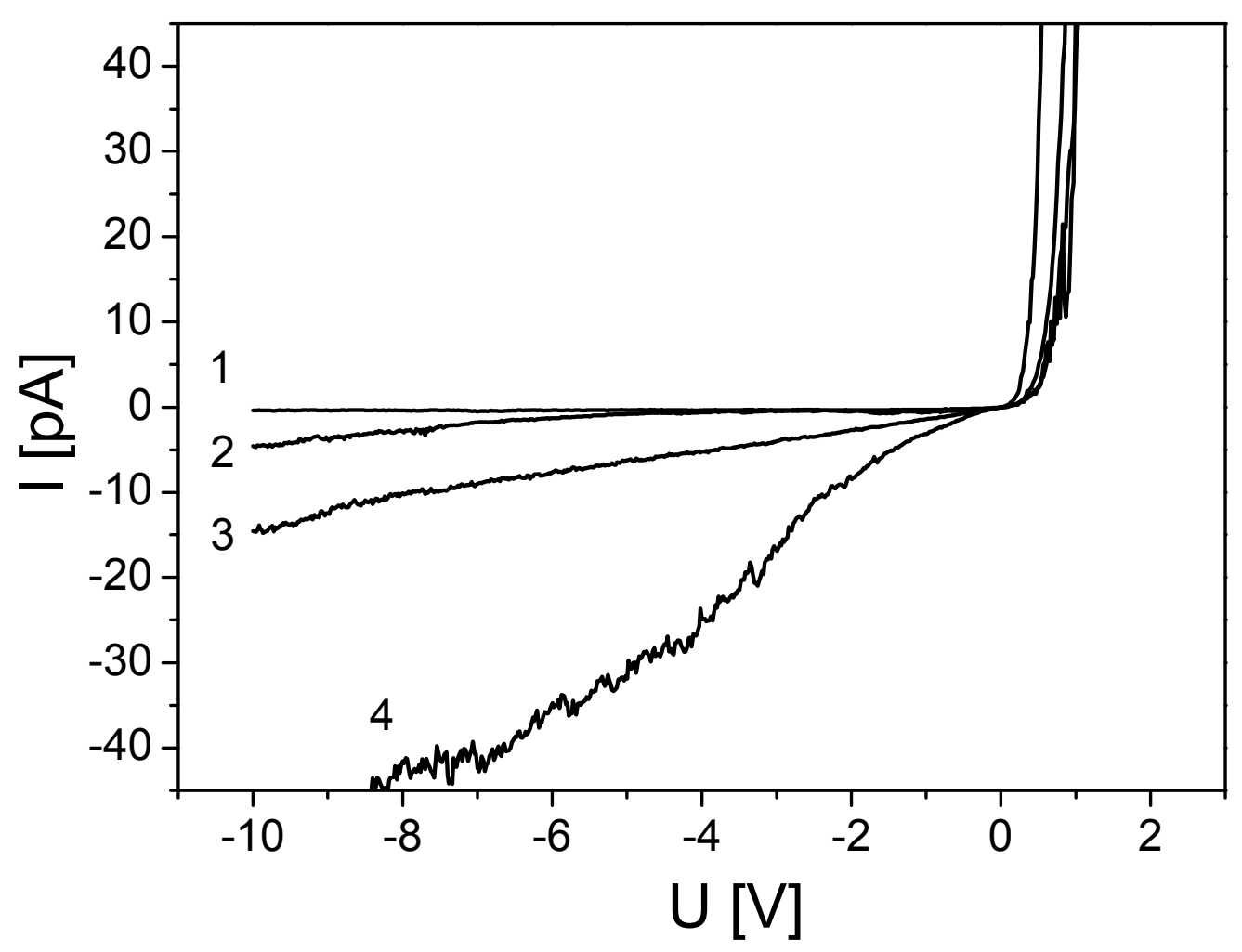

Fig. 4. Volt-ampere curves measured in various part of the aluminum islands; thickness is $<5 \mathrm{~nm}$

$$
(1), \approx 10 \mathrm{~nm}(2), \approx 15 \mathrm{~nm}(3), \text { and } \approx 20 \mathrm{~nm}(4) \text {. }
$$

A representative surface profile of a thicker aluminum film obtained by scanning tunneling microscopy is presented in Fig. 3. The spatial resolution of the surface profile is $5 \mathrm{~nm}$. The film deposition was conducted during longer time intervals (1 minute). As a result the film is thicker comparing to results on Fig. 2, also isolated islands merged to form a more homogenous bulk structure. 

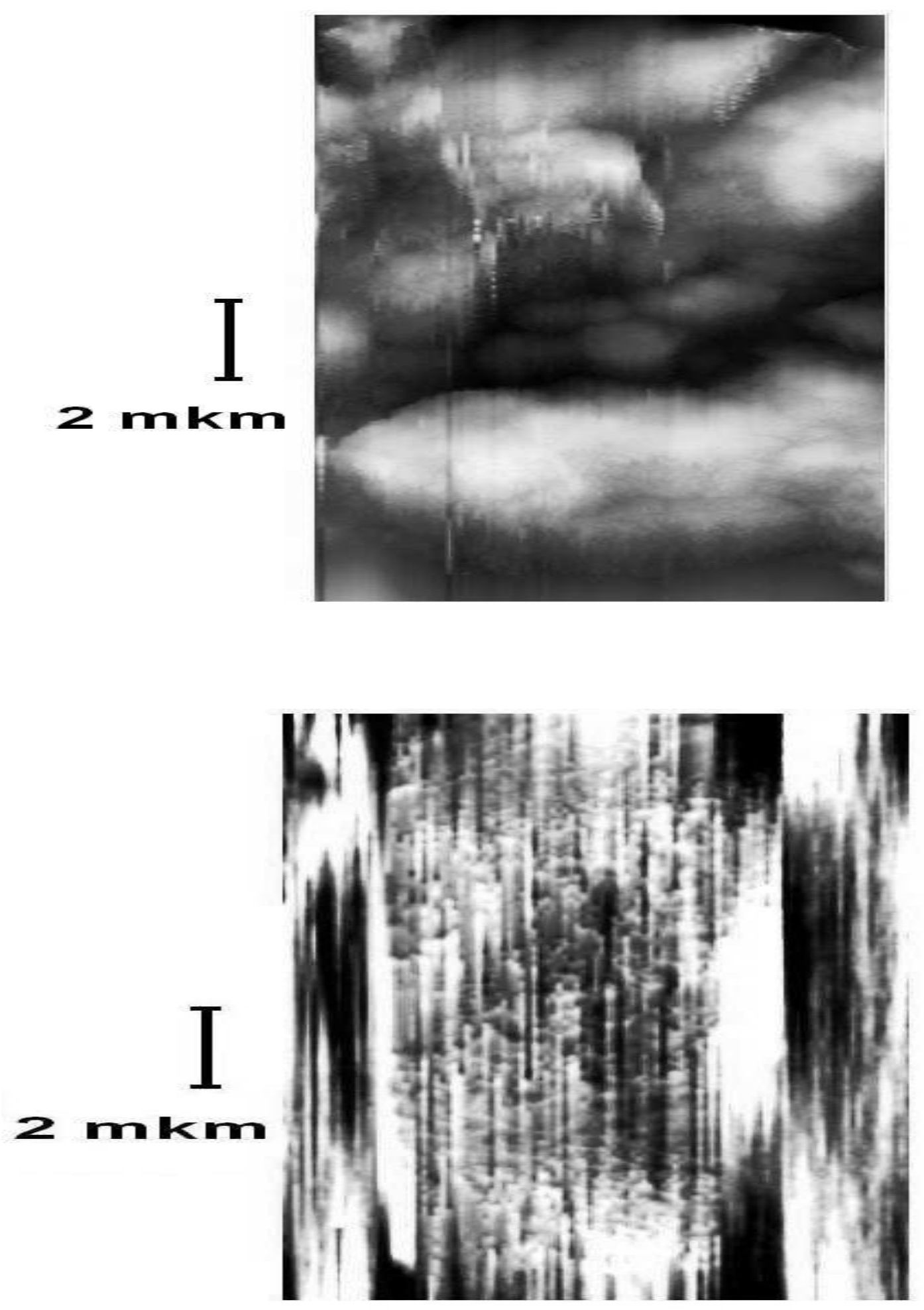

Fig. 5. (a). Kelvin probe technique, first scan results; (b). Kelvin probe technique, second scan results. 
A nanoisland volt-ampere curves are presented in Fig. 4. It characterizes both the metalsemiconductor contact phenomena and aluminum layer peculiarities. The curves do not give the island's conductivity exact numbers. First of all, the electrical current flows through the tunnelling threshold which leads to exponential decreasing of its' value depends on the threshold parameters (height and spatial characteristics). Secondly, we do not know the exact needle tip area. However the curves allow to characterize the peculiarities of current flow through a nanoisland with much quality. For example electrical currents are higher in nanoislands' thicker spots if an applied voltage had negative values. If observed as tunneling affects the metal-semiconductor border, which is a Schottky barrier, has an increase curve of slopes which is typically observable due to impurity ratios enlargement or of chemical cleaning $[19,20]$. The contact metal-semiconductor micro-characteristics can influence the volt-ampere curves of slopes in Schottky diodes [21]. However the connection between nanoislands' topological structure and volt-ampere curves of slopes has to be studied further. As we can see from the results of scanning tunnelling microscopy the nanoisland layer is representing itself a cellular porous structure with wall thicknesses in a few atomic layers. It could result in other quantomechanical effects as a result of electrical current flow through a nanowire [22]. For example, electrical current oscillations as functions of nanowires conductivity were registered in $[23,24]$. The oscillation appearance in wires with a few interatomic distance crossections were interpreted, due to aluminum peculiarities, as sp- metal. Secondly, maximum on the volt-ampere curves are originated from the nanowires parts that have different number of atoms in its' crosssection (a quantomechanical inner effect). The Fig.4 oscillations can be explained as the electrical current flows through the cellular structure walls with different crossections $(1,2, \ldots 10,11,12$.. interatomic distances). Though the electrical current flow interpretation is becoming more intricate due to nanoislands complex topology. In view of a nanoisland as an electrical scheme, the structure can be represented as a $3 \mathrm{D}$ net constructed from resistors. 
Research on thin aluminum films can be conducted by studying the alumina layers on its' surface due to its' high mechanical values and low conductivity influences which is part of aluminum properties. Scanning tunneling microscopy is not an effective way to study alumina surface because of its typical dielectrical properties. Atomic force microscopy technique allows to determine the alumina surface shape with relatively low spatial resolution. However one cannot study aluminumalumina contact layers. We believe Kelvin probe microscopy [25] provides a means to study simultaneously two surfaces: aluminum-alumina and alumina-air.

First the technique was applied on a surface that had been scanned by atomic force microscopy. Then the scanning was done at a height of 15 nanometers above the surface. During the second scan a voltage, which forced the cantilever oscillation to stop, was being measured. It gave the charge distribution on the surface. If a surface has the same potential, the second scan will measure the charge distribution profile as a plane. If the first scan determines the alumina-air surface shape, the second scan determines the aluminum-alumina contact shape. The Kelvin probe microscopy has two scans which are presented in Figs.5a,b. In order to illustrate the approach, we prepared a aluminum sample with a thick alumina layer which was obtained by extra oxidation in oxygen. As we can see from the pictures the technique allows to measure two surfaces simultaneously.

\section{Conclusions}

It was shown that, the scanning tunneling microscopy technique is quite efficient for characterization of aluminum nanoislands formed on the Si surface. The lowest layer thickness of $1 \mathrm{~nm}$ aluminum islands with rhomboidal pattern, which contacts the substrate and reflects the substrate structure, was found. The aluminum layer atoms are located along $7 \times 7$ rhombohedral unit cell on the silicon surface. The upper layers of the aluminum nanoislands possess the porous structure with face centered cubic crystal lattice. Additional information about the sample structure was obtained by mapping the scanned surface, measuring volt-ampere curves and then by comparing these data with the islands' surface shape. The volt-ampere curves measured near thicker nanoislands' parts had 
oscillations patterns which seem to be a result of a quantomechanical inner effect. The alumina and aluminum surface profiles were simultaneously registered applying Kelvin probe microscopy. The presented results can be used for development of chips with aluminum parts and devices of nanoscale integration level.

\section{References}

[1] H. Brune, K. Kern in: 1997 Physics and Chemistry of Solid Surfaces vol.8, ed. D.A. King, D.P.Woodruff (Amsterdam: Elsevier).

[2] K. Godling, R. Madden, Structure in the LII,III Absorption of Aluminum and its Oxides. Phys. Rev. 167 (3) (1968) 587-591.

[3] M. Kulawik, N. Nilius, H.-P. Rust, H.-J. Freund, Atomic Structure of Antiphase Domain Boundaries of a Thin A12O3 Film on NiAl(110), Phys. Rev. Lett. 91(25) (2003) 256101.

[4] T. Campbell, G. Aral, S. Ogata, R. Kalia, A. Nakano, P. Vashishta, Oxidation of aluminum nanoclusters, Phys. Rev. B: Condens. Matter. 71 (2005) 205413.

[5] D. Gopireddy, C. Takoudis, Diffusion-reaction modeling of silicon oxide interlayer growth during thermal annealing of high dielectric constant materials on silicon, Phys. Rev. B: Condens. Matter. 77 (2008) 205304.

[6] C.Y. Ouyang, Ž. Šljivančanin, A. Baldereschi, A First-principles study of $\gamma-\mathrm{Al}_{2} \mathrm{O}_{3}(100)$ surface, Phys. Rev. B: Condens. Matter. 79 (2009) 235410-235417.

[7] C. Ruberto, Y. Yourdshahyan, B. Lundqvist, Surface properties of metastable alumina: A comparative study of k- and $\alpha$-A12O3, Phys. Rev. B: Condens. Matter. 67 (2003) 195412.

[8] S. Mason, C. Iceman, T. Trainor, A. Chaka, Density functional theory study of clean, hydrated, and defective alumina (1102) surfaces, Phys. Rev. B: Condens. Matter. 81 (2010) 125423. 
[9] E. Wallin, J.M. Andersson, E.P. Münger, V. Chirita, U. Helmersson, Ab initio studies of Al, O, and O2 adsorption on $\alpha$-A12O3 (0001) surfaces, Phys. Rev. B: Condens. Matter. 74 (2006) 125409-1-9.

[10] P. Feibelman, Atomic arrangement and impurity bonding at a K-Al2O3 (001)/Al(771) interface: First-principles calculations, Phys. Rev. B: Condens. Matter. 76 (2007) 235405.

[11] J. Henzie, J. Barton, C. Stender, T. Odom, Large-Area Nanoscale Patterning: Chemistry Meets Fabrication, Acc. Chem. Res. 39 (2006) 249-257.

[12] T. Xu, R. Piner, R. Ruoff, An Improved Method To Strip Aluminum from Porous Anodic Alumina Films, Langmuir 19 (2003)1443-1445.

[13] B. Fischer, J. Barth, A. Fricke, L. Nedelmann, K. Kern, Growth and surface alloying of Al on $\mathrm{Au}(111)$ at room temperature, Surface Science 389 (1997) 366-374.

[14] Y. Yu, Z. Tang, Y. Jiang, K. Wu, E. Wang, Thickness dependence of the surface plasmon dispersion in ultrathin aluminum films on silicon, Surface Science 600 (2006) 4966-4971.

[15] J. Pierce, N. Bartelt, R. Stumpf and K. McCarty, Stability of ultrathin alumina layers on NiAl(110), Phys. Rev. B: Condens. Matter. 77 (2008) 195438.

[16] R. Lazzari, J. Jupille, Wetting and interfacial chemistry of metallic films on the hydroxylated a-A12O3,,0001... surface, Phys. Rev. B: Condens. Matter. 71 (2005) 045409.

[17] A. Olivaa, P.O. Quintanaa, J. Ceha, M. Coronaa, Aguilarb 1999 Current induced effects in aluminum thin films, Thin Solid Films 353 (1999) 1-7.

[18] G. Binnig, H. Rohrer, Ch. Gerber, E. Weibel, 7x7 Reconstruction on Si(111) Resolved in Real Space, Phys. Rev. Lett. 50(2) (1983) 120-123.

[19] B.L. Sharma, Metal-Semiconductor Schottky Barrier Junctions and Their Applications, Plenum Pub Corp, New York, 1984.

[20] Y. Hirota, 1993 Schottky characteristics of GaAs surface cleaned by ultrasonic running deionized water treatment. Appl. Phys. Lett. 63(14) (1993) 1936-1938. 
[21] L. Lajaunie, M. David, J. Barbot, Physical properties of Co/n-Ge Schottky contacts. J. Phys. D: Appl. Phys. 4 (2011) 125103.

[22] N. Agrait, A. Yeyati, J. van Ruitenbeek, Quantum properties of atomic-sized conductors, Physics Reports, 377 (2003) 81-279.

[23] B. Ludoph, J. van Ruitenbeek, Conductance fluctuations as a tool for investigating the quantum modes in atomic-size metallic contacts, Phys. Rev. B: Condens. Matter. 61 (2000) 2273-2285.

[24] R. Smit, Y. Noat, C. Untiedt, N. Lang, M. van Hemer, J. van Ruitenbeek, Measurement of the conductance of a hydrogen molecule, Nature 419 (2002) 906-909.

[25] F. Miller, A. Vandome, J. McBrewster, Kelvin Probe Force Microscope, Berlin: Betascript Publishing, 2010. 